\title{
Reconstruction of corneal epithelium with cryopreserved corneal limbal stem cells in a goat model
}

\author{
Shengli Mi ${ }^{1}$, Zhongying Dou ${ }^{1}$, Qingmei Zhao ${ }^{1}$, Xueyi Yang ${ }^{1}$, Lei Qu ${ }^{1}$, Shuming Chen ${ }^{1}$, Jinlian Hua ${ }^{1}$ \\ ${ }^{l}$ Northwest university of A\&F, Shaanxi Branch of National Stem Cell Engineering and Technology Centre, Yangling, China
}

This paper reports a procedure to construct an artificial corneal epithelium from cryopreserved limbal stem cells for corneal transplantation. The limbal stem cells were separated from limbal tissue of male goats, the 2 to 3 passages of limbal stem cells identified by flow cytometric analysis were cryopreserved in liquid nitrogen. The cryopreserved corneal limbal stem cells were thawed and examined with trypan blue staining for their viability, then transplanted onto the top of a cell-free human amniotic membrane framed on a nitrocellulose sheet and grown for 12-14 days in culture medium to construct corneal epithelium sheets. The corneal epithelium thus generated from limbal stem cells was transplanted into the right eye of pathological models of total limbal stem cells deficiency(LSCD) that had been created in 10 female goats. Another 6 model female goats, including 3 just transplanted with cell-free amniotic membrane and 3 only with injured tissue resected, were designed as control. Then the clinical observation and histological examination were used to evaluate the effects of the reconstruction of corneal epithelium. Polymerase chain reaction analysis was used for detection of the SRY gene to identify whether male goat limbal cells exist in the female goats for a long time. During the six-month recovery period, we found that the corneal transparency of the experimental goats was significantly improved in comparison with that of control goats. The SRY gene of the male goat could be found in one female eye after transplantation. The results showed that the cryopreserved corneal limbal stem cells are able to repair the damaged goat cornea, suggesting a new clinical approach to reconstruct the corneal epithelium.

Keywords: tissue engineering, limbal stem cell, Amniotic membrane, transplantation

Cell Research (2008) 18:s73. doi: 10.1038/cr.2008.163; published online 4 August 2008

Correspondence: Shengli $\mathrm{Mi}^{\mathrm{a}}$, Zhongying Dou ${ }^{\mathrm{b}}$

a'E-mail: shenglimi@yahoo.com.cn

${ }^{\mathrm{b}}$ E-mail: douzhongying@china.com 